\title{
ANÁLISE DESCRITIVA DO NÍVEL DE STRESS EM PARTICIPANTES DE UM PROGRAMA DE ORIENTAÇÃO E REEQUILÍBRIO POSTURAL EM GRUPO
}

\author{
Débora Fernandes dos Santos, Jessica Jessica Ribeiro, Letícia Endsfeldz Teixeira, Célia Aparecida \\ Stellutti Pachioni, Dalva Minonroze Albuquerque Ferreira, Ana Luiza Pires Batista
}

Curso de Fisioterapia da Universidade Estadual Paulista Júlio de Mesquita Filho - Faculdade de Ciências e Tecnologia FCT/UNESP. E-mail: fisiofernandes@hotmail.com

\section{RESUMO}

Cada vez mais, pessoas apresentam sinais de stress que pode ser caracterizado como uma situação de tensão que produz mudança no comportamento físico e emocional do indivíduo, e uma forma de minimiza-lo é a atividade física. O objetivo do estudo foi avaliar o nível de stress em participantes do programa de orientação e reequilíbrio postural em grupo da Faculdade de Ciências e Tecnologia da UNESP - Campus de Presidente Prudente. O estudo transversal analisou 12 sujeitos de ambos os sexos (56 \pm 19 anos) que realizam regularmente o programa. Para a avaliação do stress dos participantes foi utilizado o Inventário De Sintomas De Stress Para Adultos De Lipp. Os resultados demonstraram que $25 \%$ dos participantes apresentaram elevação no nível de stress e $75 \%$ não apresentaram nenhuma alteração. O programa se torna um grande colaborador no enfoque da prevenção do nível ou índice de stress de seus participantes.

Palavras-chave: Stress, Qualidade de Vida, Reequilíbrio postural; Atividade Física; Bem - estar.

\section{INTRODUÇÃO}

Desde a segunda metade do século XX tem-se presenciado uma transformação no padrão de vida dos seres humanos. Com a mecanização e os avanços tecnológicos houve redução da realização de atividade física, adotados como forma de poupar esforços. Paralelamente a isso, ocorreram mudanças nos padrões alimentares, criando um ambiente propício para a exposição ao stress e diminuição da qualidade de vida de grande parte da população (NAHAS, 2000).

No Brasil, o nível de stress aumentou significativamente nos últimos anos; cada vez mais pessoas apresentam sinais desse problema. Uma pesquisa realizada pela International Stress Management Association (ISMA-BR) aponta o Brasil, como o segundo pais onde o stress é mais relatado, sendo 40\% na população de São Paulo (LIPP, 2004). Segundo Selye (1936), o stress é uma reação do organismo que ocorre frente a situações que exijam dele adaptações além do seu limite.

O stress pode ser caracterizado como uma situação de tensão aguda ou crônica que produz uma mudança no comportamento físico e no estado emocional do indivíduo, sendo uma resposta de adaptação psicofisiológica que pode ser negativa ou positiva dependendo do organismo 
(MOLINA, 1996), ao que se refere à relação entre o corpo e a mente (LEWIS, 1988; CERCHIARI, 2000; LEAL, 2001).

Estudos sobre o stress, além de abordar as consequências relacionadas ao corpo e mente do homem, abrangem as implicações desse problema na qualidade de vida da sociedade, sendo assim, o stress pode afetar a saúde, a qualidade de vida e a sensação de bem-estar como um todo (LIPP, 2001).

O excesso de stress, além do conhecido desgaste físico e/ou mental, leva ao envelhecimento precoce, uma série de doenças e até a morte (LIPP, 1998), além de prejudicar o desempenho e as atividades diárias do indivíduo, gerando desconfortos, cansaço e diminuindo seu ritmo e capacidade de manter uma vida equilibrada e saudável.

Uma das consequências acarretadas pelo stress acorre através das tensões musculares que causam respostas negativas ao corpo humano, sendo uma delas os desvios posturais ou posturas viciosas, que provocam desconfortos e dores ao corpo.

A atividade física é reconhecida como um dos métodos mais simples e eficazes para reduzir o stress, porque o corpo retorna ao equilíbrio natural, dando sensação de relaxamento ao indivíduo (DAVIS, 1996).

Uma forma de atividade física, que atua como tratamento para minimizar o stress e as consequências relacionadas ao corpo, são os trabalhos de orientação e reequilíbrio postural em grupo, que objetiva envolver consciência corporal, a reeducação respiratória, melhorar e orientar os maus hábitos posturais. Por ser um tratamento em grupo, vem de encontro à dinâmica de socialização com outros indivíduos que não são do seu convívio diário, ou seja, relacionado ao componente estressor, trazendo um bem estar, por meio da realização da pratica de atividade física.

O objetivo deste estudo foi avaliar o nível de stress em participantes do programa de orientação e reequilíbrio postural em grupo da Faculdade de Ciências e Tecnologia da FCT/UNESP - Campus de Presidente Prudente

\section{METODOLOGIA}

Trata-se de um estudo transversal com 12 participantes, de ambos os gêneros que realizam regularmente o programa de orientação e reequilíbrio postural em grupo. As atividades do programa são realizadas duas vezes por semana com duração de 60 minutos cada uma. 
O projeto de extensão universitário intitulado “Programa de orientação e reequilíbrio postural em grupo (PORPOG)", foi aprovado pelo Comitê de Ética em Pesquisa da FCT/UNESPCampus de Presidente Prudente, através do Processo no 194/2007, e desde então é desenvolvido no Centro de Estudos e Atendimentos de Fisioterapia e Reabilitação (CEAFIR) da FCT/UNESP Campus de Presidente Prudente. O programa consta de alongamento, fortalecimento, equilíbrio, coordenação e conscientização corporal, além de orientar quanto aos maus hábitos posturais, corrigir e prevenir posturas inadequadas durante as atividades, promover a conscientização da postura e favorecer o relacionamento social em grupo.

Para a avaliação do stress dos participantes foi respondido o Inventário De Sintomas De Stress Para Adultos De Lipp, que identifica a sintomatologia que o indivíduo apresenta, avaliando se o mesmo apresenta sintomas de estresse, o tipo de sintomas existentes (se somáticos ou psicológicos) e a fase em que se encontra (alerta, resistência, quase exaustão e exaustão). 0 questionário é composto por três quadrantes, sendo o primeiro referente aos sintomas experimentados pelo indivíduo nas últimas 24 horas (15 questões), o segundo quadrante dos sintomas da última semana (15 questões), e o terceiro aos sintomas do último mês (23 questões) (LIPP, 2000).

A correção e interpretação do "Inventário De Sintomas De Stress Para Adultos De Lipp" foi realizada pela psicóloga Ana Luiza Pires Batista (CRP 06/15792), de acordo com as diretrizes do Conselho Federal de Psicologia quanto ao uso de testes.

\section{RESULTADOS}

A amostra foi constituída por 12 indivíduos, destes, dez do gênero feminino (83,33\%) e dois do gênero masculino (16,66\%), com idade média de $56 \pm 19$ anos.

Dentre os resultados verificaram que sete indivíduos (58,33\%) são aposentados, três indivíduos (25\%) são professores, um (8,33\%) é estudante e um (8,33\%) é assistente social. Em relação ao stress, apenas três indivíduos (25\%) foram classificados como estressados e nove (75\%) não foram classificados como estressados.

Quanto ao nível de estresse, foi constatado que dentre os $25 \%(n=3)$ dos indivíduos, estão na fase de alerta $16,6 \%(n=2)$, já $8,33 \%(n=1)$ está na fase de exaustão (Tabela 2 ). 
Tabela 2. Níveis de estresse dos participantes do Projeto de orientação e reequilíbrio postural em grupo.

\begin{tabular}{lcc}
\hline & $N^{\circ}$ de participantes & Participantes \\
\hline Apresentam stress & 3 & $25 \%$ \\
Fase de resistência & 2 & $16,6 \%$ \\
Fase de exaustão & 1 & $8,33 \%$ \\
\hline
\end{tabular}

E ainda, dentre os indivíduos que apresentam stress, cada um apresenta um tipo de sintomatologia diferente, ou seja, sintomas psicológicos, sintomas físicos e um indivíduo ambos os sintomas.

\section{DISCUSSÃO}

O stress tem sido muito estudado por ser um problema que gera preocupações pelas graves consequências que pode acarretar na qualidade de vida do homem; assim como citado por Lipp, Malagris e Novaes (2007), o stress pode desencadear sérios prejuízos na saúde da pessoa.

Considerando o ritmo acelerado das transformações ocorridas na sociedade moderna, como citado anteriormente, o homem precisa adaptar-se de maneira rápida a estas mudanças, evitando o surgimento de doenças advindas dos altos níveis de estresse. Apesar das mudanças citadas, que levam o indivíduo cada vez mais a ultrapassar seu limite físico e psicológico, neste estudo podemos observar uma diferença em relação a outros estudos, onde a maioria dos participantes (75\%) se apresentou sem stress, pelo fato de alguns deles participarem a algum tempo do grupo.

Segundo Oliveira (2004) alguns fatores externos ao stress estão ligados à ocupação profissional exercida; e para Carayon, Smith \& Haims (1999); Dejours, Dessors \& Desriaux (1993); Schabracq, Winnubst \& Cooper (1996); Seligmann-Silva (1994), além dos fatores externos, as exigências físicas e mentais da atividade (organização do trabalho) podem estar entre os estressores responsáveis pelo stress decorrente do trabalho. Sendo essa, uma possível explicação para o baixo nível de stress presente em nossa amostra já que, mais da metade dos indivíduos (58,33\%) são aposentados.

Atualmente, atividade física pode ser entendida como qualquer movimento corporal, produzido pela musculatura esquelética, que resulta em gasto energético, tendo componentes e determinantes de ordem biopsicossocial, cultural e comportamental, podendo ser exemplificada por danças, esportes, exercícios físicos, atividades laborais e deslocamentos. A atividade física é de grande importância, pois atua no controle do stress possibilitando que o indivíduo utilize o excesso 
de adrenalina produzido em situações de tensões e reduza a ansiedade decorrente da ocupação (PITANGA, 2002).

O que corrobora com nosso estudo, já que um elemento de importante influência no resultado apresentado é o fato dos participantes realizarem regularmente o programa de orientação e reequilíbrio postural em grupo, de acordo com Sadir (2010) onde demonstra que a implantação de programas que promovam condições para a promoção da saúde física e emocional das pessoas, como a atenção que deve ser dada ao stress, tendo em vista todas as suas possíveis consequências, mostra-se benéfica e necessária.

\section{CONCLUSÃO}

Os participantes do programa de orientação e reequilíbrio postural em grupo não apresentaram alterações no nível de stress.

Pensando na melhora da qualidade de vida, e na minimização dos sintomas de stress, o programa de orientação e reequilíbrio postural em grupo se torna um grande colaborador no enfoque da prevenção da saúde mental e favorece o bem-estar físico e mental da população.

\section{REFERÊNCIAS}

1. NAHAS, M. V., BARROS, M. V. G, FANCALACCI, V. O Pentáculo do bem-estar - Base Conceitual Para Avaliação do Estilo de Vida de Indivíduos ou Grupos. Atividade Física \& Saúde, v.5, 2, 2000.

2. LIPP, M. E. N. Stress no Brasil: Pesquisas avançadas. Campinas: Ed. Papirus, 2004.

3. SELYE, H. A. Syndrome produced by diverce nervous agents. Nature, 138, 32, 1936. http://dx.doi.org/10.1038/138032a0

4. MOLINA, O. F. Estresse no cotidiano. São Paulo: Pancast, 1996.

5. LEWIS, M. E. \& LEWIS, H. R. Fenômenos Psicossomáticos: até que ponto as emoções podem afetar a saúde. Rio de Janeiro: José Olimpio editora, 1988.

6. CERCHIARI, E. Psicossomática: um estudo histórico e epistemológico. Revista de Psicologia: ciência e profissão. 20(4), pp.64-79, 2000.

7. LEAL, E. Q. Stress em adolescentes: Avaliação dos principais sintomas. Universidade Federal da Paraíba. Mestrado em Ciências do Desenvolvimento Humano, Centro de Ciências da Saúde. Paraíba, 2001.

8. LIPP, M. E. N. Pesquisas sobre stress no Brasil: Saúde, ocupações e grupos de risco. Campinas:Papirus, 2001. 
9. LIPP, M.E.N. Como Enfrentar o Stress. 5. ed. São Paulo: Ícone, 1998.

10. DAVIS, M.; ESHELMAN, E. R.;MCKAY, M. Manual de relaxamentp e redução do stress. São Paulo, S.P: Summus, 1996.

11. LIPP, M. E. N. Inventário de sintomas de stress para adultos de Lipp (ISSL). São Paulo: Casa do Psicólogo, 2000.

12. LIPP, M. E. N., MALAGRIS, L. E. N., \& NOVAIS, L. E. Stress ao longo da vida. São Paulo: Ícone, 2007.

13. OLIVEIRA, J. B. Fontes e sintomas de stress em juízes e servidores públicos: Diferenças entre homens e mulheres. Dissertação de Mestrado não-publicada, Pontifícia Universidade Católica de Campinas, Campinas, SP, 2004.

14. CARAYON, P., SMITH, M.J. \& HAIMS, M.C. Work organization, job stress, and workrelated musculoskeletal disorders. Human Factors, 41, 644-663, 1999. http://dx.doi.org/10.1518/001872099779656743

15. DEJOURS, C., DESSORS, D. \& DESRIAUX, F. Por um trabalho, fator de equilíbrio. RAE Revista de Administração de Empresas,33(3), 98-104, 1993. http://dx.doi.org/10.1590/S0034$\underline{75901993000300009}$

16. SCHABRACQ, M. J., WINNUBST, J. A. M., \& COOPER, C. L. Handbook of Work and Health Psychology. London: John Wiley \& Sons, 1996.

17. SELIGMANN-SILVA, E. Desgaste Mental no Trabalho Dominado. Rio de Janeiro: Editora UFRJ, 1994.

18. PITANGA, F. J. G. Epidemiologia, atividade física e saúde. Rev. Bras. Ciên. e Mov. Brasília v.10 n. 3 p. julho 2002.

19. SADIR, M. A., BIGNOTTO, M. M., \& LIPP, M. E. N. Stress e qualidade de vida, 2010. 